\title{
Research at the University of Kent and Subsequent Research Activities
}

\author{
Kyriacos KALLI \\ Nanophotonics Research Laboratory, Department of Electrical Engineering and Information Technology, \\ Cyprus University of Technology, Limassol, 3036, Cyprus \\ *Corresponding author: Kyriacos KALLI_Ｅ-mail: Kyriacos.Kalli@cut.ac.cy
}

\begin{abstract}
The author's research activities undertaken at the Applied Optics Group, the University of Kent at Canterbury are reviewed, during his time there from 1988-1992 and 1994-1996, followed by a summary of recent research. The areas of interest are high finesse ring resonators, tunable optical filters, novel optical fiber grating sensors in glass and polymer, femtosecond laser inscription and micromachining, environmental pollution monitoring, hydrogen activated $\mathrm{Pd}$ films on silicon and impurity measurement on silicon wafers.
\end{abstract}

Keywords: Ring resonators, fiber gratings, tunable optical filters, femtosecond laser, pollution monitoring, thin films, silicon

\section{Introduction}

I arrived at the University of Kent in 1985 as an undergraduate student studying theoretical physics, and on completion of my degree chose postgraduate studies that were applied in nature. Thus I began a diverse 23-year exploration of the optical science that is continuing to this day. Other than the years at Kent as student, researcher and educator, I have spent time at the Fiber and Electro-Optics Research Center, Virginia Tech. (1993), the University of Cyprus (1997-2001), the Higher Technical Institute (2001-2008), and I am currently an academic at the Cyprus University of Technology. I have also had the opportunity to be a long-term visiting fellow with the Photonics Research Group at Aston University (2002 onwards). Much of my time has been spent studying fiber optic devices and sensors, whereas my time in Cyprus began with work in the field of solid-state physics.

\section{2. $\mathrm{PhD}$ research at Kent}

\section{Optical fiber ring resonators}

I will begin chronologically highlighting first the work undertaken in my doctoral thesis [1], through the years 1988-1992, studying linear and non-linear phenomena in high finesse optical fiber ring resonators. This proved a challenging project as I manufactured the ring resonators from a continuous piece of optical fiber that was machine polished at two points to make evanescent wave coupler halves that when joined, having looped the fiber, constituted the optical resonator (Fig. 1).

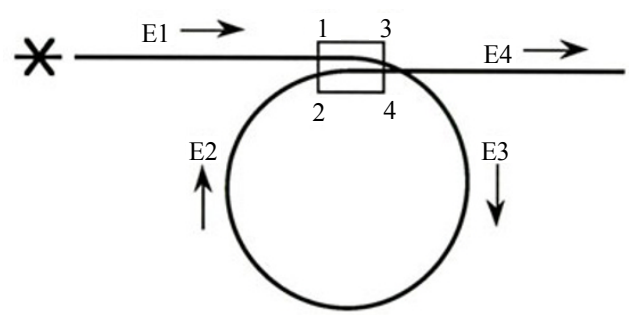

Fig. 1 Schematic of the all fiber ring resonator.

Received: 17 June 2011 / Revised version: 22 June 2011

(C) The Author(s) 2011.This article is published with open access at Springerlink.com 
It was critical that both coupler halves were manufactured to very high quality, such that optical contact bonding was possible, and that they were of very low loss. In this way the dominant loss factor was the inherent loss of the optical fiber. Initially devices were made to operate at $633 \mathrm{~nm}$, but we soon realized that by producing devices to work in the low loss telecommunications windows of $1300 \mathrm{~nm}$ and $1500 \mathrm{~nm}$ we could create very long resonant cavities that would offer extremely high optical resolution, if they were accompanied by high finesse (Fig. 2).

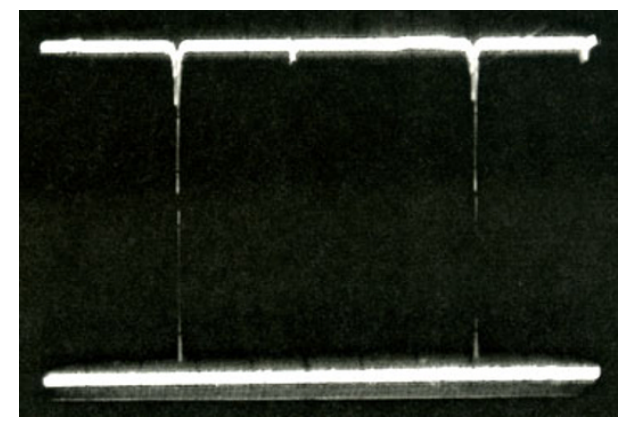

Fig. 2 High finesse output at $633 \mathrm{~nm}$ shows a highly symmetric transmission response for a finesse of 400 .

At that time there was much interest in producing multiple-beam, resonant fiber devices with enhanced phase sensitivity compared with two-beam interferometers. Furthermore, it was recognized that the resonator design constituted a high gain, optical cavity that was well suited to generating non-linear processes such as Brillouin scattering, principally because the low loss design allowed for a very low threshold for optical non-linearity (Fig. 3).

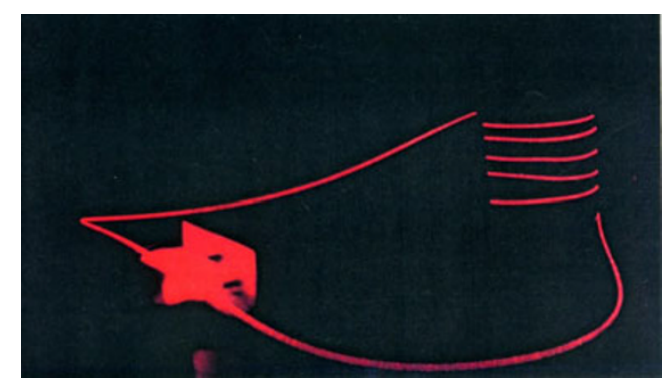

Fig. 3 Scattered light from a ring resonator operating at $633 \mathrm{~nm}$, when held at resonance, and the cavity gain is about 40 .

This allowed for the development of novel fiber lasers that did not require a doped section of fiber to act as the optical gain medium, but rather exploited the presence of the intrinsic acoustic phonons in the fiber to behave as an effective gain medium.

Two or more Brillouin signals mixed from one or more ring resonators were used to realize novel frequency and microwave signal generators [2-4]. The extremely high finesse, with the long cavity lengths of up to $40 \mathrm{~m}$, allowed for filtering of signals directly in the optical domain, thereby offering unambiguous measurement of laser line-widths in the $\mathrm{kHz}$ range [5], with implications for laser Doppler velocimetry measurements (Fig.4) [6]. We demonstrated the potential of the ring resonator as an extremely high resolution optical spectrum analyzer, directly measuring the frequency difference of two laser lines to better than $20 \mathrm{kHz}$. This was a record achievement at that time.

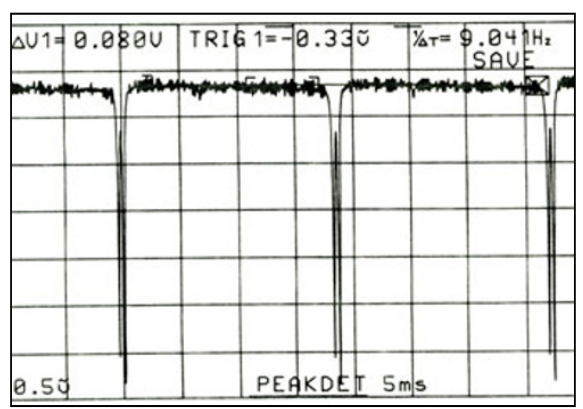

Fig. 4 Ring resonator spectrum of two single frequency lasers with a frequency difference of $100 \mathrm{kHz}$.

A new type of tunable fiber frequency shifter that utilized the high optical resolution to select discrete frequency components of a frequency modulated light beam, whilst offering very high suppression of unshifted light and unwanted signal sidebands, was shown to be an effective alternative to more traditional devices [7]. We also investigated, experimentally and theoretically, the dynamic response of the ring resonators to frequency swept input signals that varied on a time scale comparable to the cavity response time, for which the resonator finesse was required to reach its steady-state value (Fig. 5) $[8,9]$. 

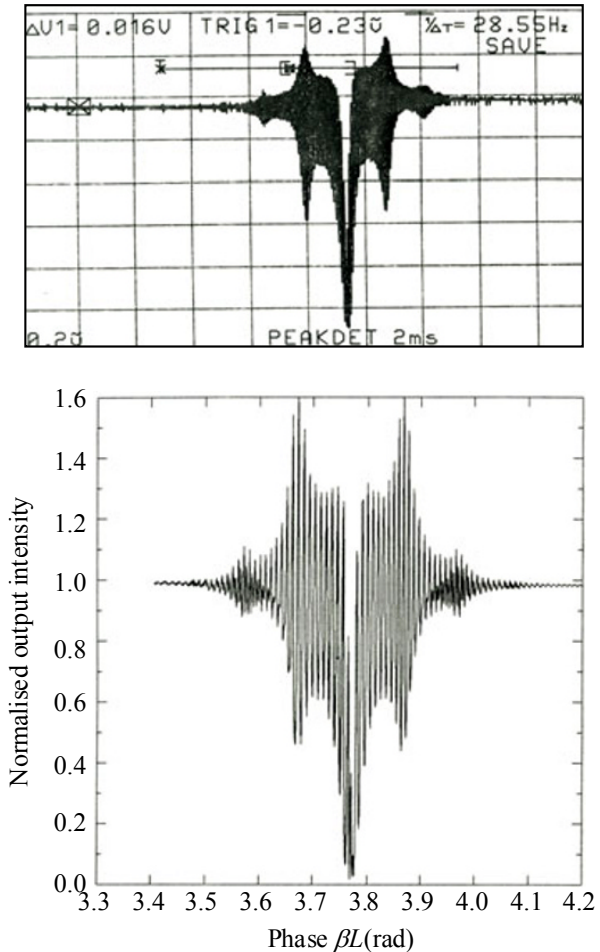

Fig. 5 Ring resonator filtered spectrum of a phase modulated signal at $100 \mathrm{kHz}$ (top), relevant theoretical curve (bottom).

New types of gyroscopic sensors were developed that were based on the concept of low coherence multiplexing, as a means to yield improved sensitivity when compared with the conventional two-beam Sagnac interferometer [10]. Here the coherence length of the source is short in comparison with the loop length of the ring resonator and there is no build-up of finesse or optical interference at the output of the single interferometer. However, the interferometer can be designed to be balanced and reciprocal in nature for particular path pairs that consist of single and multiple circulations of clockwise and counterclockwise beams. Interference between beams traversing the virtually identical optical paths results in an output that is a summation of individual average intensities of the recirculating fields and this can offer advantages in sensor sensitivity. However, a consequence of these multiple recirculating fields is an increase in phase induced intensity noise, which results from non-zero correlations among the various intensity terms of the recirculating fields [1].

\section{A second period at Kent}

\subsection{Bragg sensor demodulation}

I later returned to Canterbury through the years 1994-1996. This was a particularly dynamic time in the field of optical fiber sensors based on fiber Bragg gratings (FBG), and there was enormous interest in methods that were capable of demodulating the perturbation-induced wavelength shift output of the sensors, but without the use of bulky and expensive optical spectrum analyzers [11-13]. Furthermore, real world applications demanded that multiple sensors could be demodulated using efficient methods ideally with a single source and detector, respectively illuminating and recovering data from a complex optical network.

Some of the research was focused on extending the FBG "sensor count" by novel wavelength demodulation techniques, whereas other work was focused on using gratings to tailor the network performance. I will begin by discussing the use of chirped gratings for the stable synthesis of dual wavelength sources by reflecting different wavelength bands of a superfluorescent fiber source; this is based on the classic dual wavelength method [14]. The spectral content of the synthesised wavelengths allowed for the generation of two low coherence sources that were ideal for use with a coherence tuned network, allowing for passive, remote sensing. The sensor network consisted of a Fizeau cavity, coherence tuned to a Mach-Zehnder interferometer, for which we demonstrated an unambiguous sensing range of 270 microns with a dynamic range of $2.7 \times 10^{5}$. The advantage in using chirped gratings for the dual wavelength synthesis results from more of the net source power being reflected by the gratings compared with single, narrow band gratings. Moreover, with this approach a temperature induced shift in the grating center wavelength results in a ten times smaller shift in the synthesised source mean wavelengths, compared with narrow band FBG. This is important, as for a 
practical sensor the extended phase measurement is critically dependent on the stability of the synthesised wavelengths.

We demonstrated the first instrumentation for the simultaneous interrogation of two-beam interferometric and Bragg sensors [15]. The technique employed an unbalanced Mach-Zehnder interferometer illuminated by a single low-coherence source, acting as a wavelength tunable source for the grating and as a path-matched filter for a Fizeau interferometer, thus providing a high phase resolution output for each sensor.

The demultiplexing of large sensor arrays suitable for low frequency signal interrogation was investigated. In the first approach a series of eight FBG were demultiplexed, using an unbalanced Mach-Zehnder interferometer illuminated by a single low-coherence source that provided a high-phase-resolution output for each sensor [16].

The outputs were sequentially selected in wavelength by a tunable Fabry-Perot interferometer, giving a minimum detectable strain of $90 \mathrm{n} \varepsilon-\sqrt{\mathrm{Hz}}$ at $7 \mathrm{~Hz}$ at $1535 \mathrm{~nm}$ (Fig. 6). Arrays of up to $32 \mathrm{Bragg}$ grating strain and temperature sensors, with identical characteristics, were demodulated using a prototype system based on interferometric wavelength-shift detection, for measuring quasi-static strain and temperature [17].

I close this section on early Bragg grating work by noting that we also demonstrated a novel method to separate strain and temperature induced wavelength shifts using a single Bragg grating. The approach is based on the fact that the refractive index change of the grating is not purely sinusoidal. This leads to a Bragg reflection at the first $(\sim 1500$ $\mathrm{nm})$ and second $(\sim 800 \mathrm{~nm})$ diffraction orders of the grating; measurements at these wavelengths may be used to determine the wavelength dependent strain and temperature coefficients, from which independent temperature and strain measurements can be recovered [18].

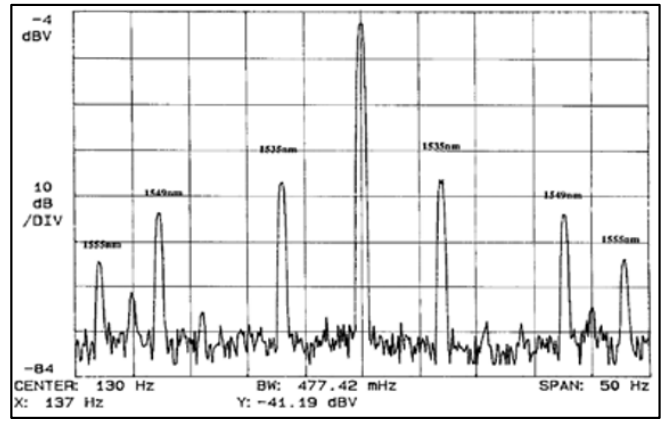

(a)

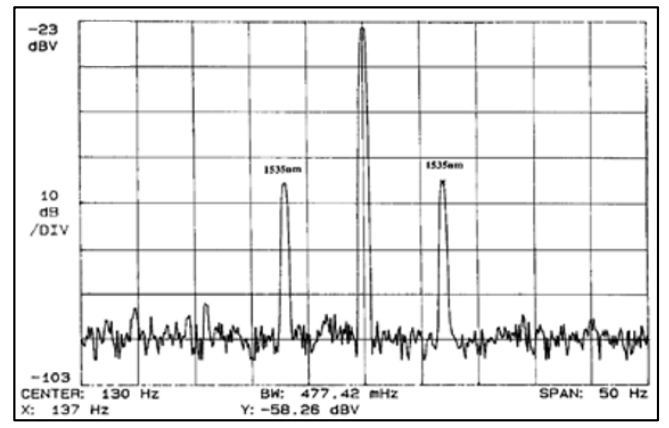

(b)

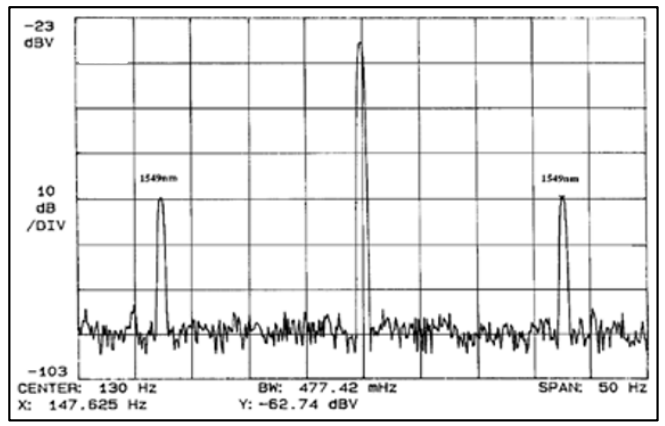

(c)

Fig. 6 (a) Power spectrum indicating the presence of multiple signals reflected from gratings at $1535 \mathrm{~nm}, 1549 \mathrm{~nm}$, and $1555 \mathrm{~nm}$ in the absence of the tunable Fabry-Perot filter, and superimposed upon a $130 \mathrm{~Hz}$ carrier generated by the Mach-Zehnder interferometer, (b) and (c) spectra indicating how the filter allows for discrete wavelength selectivity, as it is tuned to sensor $1(1535 \mathrm{~nm})$ and sensor 2 (1549 nm), respectively.

\subsection{Raman spectroscopy for pollution monitoring}

Finally, I was sponsored by Shell Research to undertake study into the use of optical spectroscopy, specifically Raman spectroscopy, for the measurements of volatile organic compounds in ground water [19]. There was, and continues to be, great interest in this research field, as ageing storage 
facilities pose the danger of leaking petroleum into the ground, with the potential contamination of the water table. Interesting aspects to this research were the design of new optical probes for collecting the Raman signal remotely using miniature optics coupled to optical fibers, connected to small-scale spectrometers - in this way remote sensing was possible. Finally, we investigated the use of polymer concentrators to improve the effective sensitivity of the system by selectively extracting the poly-aromatic hydrocarbons from water.

\section{Research between and after the Kent years}

My time at Virginia Tech. was principally based on industrial research projects in the following areas. Firstly, the development and testing of extrinsic/intrinsic low-finesse fiber Fabry-Perot interferometers as temperature monitors on board naval vessels [20]: both hybrid sapphire rod/optical fiber sensors (extrinsic) and gold-coated optical fiber sensors (intrinsic) were successfully tested to $300{ }^{\circ} \mathrm{C}$ and $1000{ }^{\circ} \mathrm{C}$, respectively. Secondly, the development of an optical fiber intruder detection system, based on a Sagnac loop, was successfully tested in the laboratory. Information was extracted from the path dependent phase sensitivity of the Sagnac interferometer and by examination of the frequency histogram generated by the "intruder". Finally, I studied finite and infinite impulse response filters for signal processing directly in the optical domain. The filters were based on Mach-Zehnder and ring resonator networks, with and without optical gain.

An extension of the first work in environmental pollution monitoring was an EU-sponsored project RAMFLAB, which I undertook at the University of Cyprus, in collaboration with Applied Optics Group at the University of Kent, IROE-CNR Firenze, Italy and Tel-Aviv University, Israel. Here the concentration of several pollutants, usually present in industrial waste water, was measured. Data was modeled using neural network data processing of absorption and fluorescence measurements in the visible spectral range. A result of appropriate network training provided quantitative analysis of many pollutants with sub-ppm resolution. We used compact optical fiber instrumentation for absorption spectroscopy and an innovative flow-cell for the fluorescence measurements, with both setups enabling cost-effective, in situ, nonstop monitoring of waste water [21].

Before moving onto recent research activity, I conclude this section with a discussion of research into hydrogen activated $\mathrm{Pd}$ films on silicon and polyvinylidene fluoride (PVDF) and impurity measurements on silicon wafers, also undertaken at the University of Cyprus [22-24].

The processes employed to clean silicon wafers can, paradoxically, be a major source of impurities. This is particularly so in the case of metallic contamination, and therefore wafer-monitoring techniques have grown in importance. Any detection method must be non-destructive and non-contacting whilst offering a high spatial resolution. We used photothermal radiometry (PTR) as a non-destructive evaluation methodology, measuring the blackbody radiation emitted from a material excited by a modulated laser source [25]. Information is recovered regarding the electronic and thermal properties of the semiconductor as a function of the laser modulation frequency. An ideal blackbody radiator produces a linear frequency spectral response. In contrast, implanted or doped silicon wafers have a frequency spectrum that is biased at lower frequencies from the thermal component (Fig. 7), and at higher frequencies by the electronic material properties (Fig. 8).

We have shown that the degree of the relative contributions gives information that is dependent on the type of doping and the material characteristics. Indeed information on both frequency and time dependent behavior of metal contaminated silicon wafers is recovered that may prove useful in the wafer manufacturing process. 


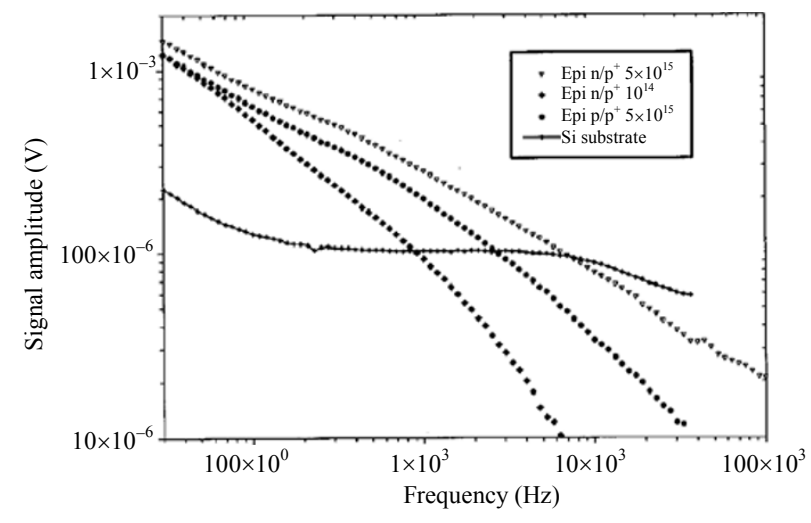

Fig. 7 PTR signal vs modulation frequency for contaminated silicon wafers, displaying clear thermal material behavior and compared to a standard silicon substrate.

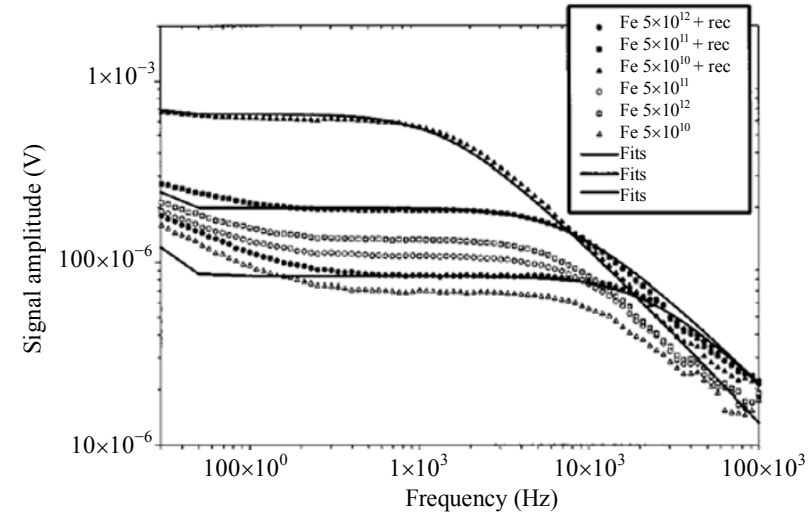

Fig. 8 PTR signal vs modulation frequency for contaminated silicon wafers, displaying clear electronic material behavior with fitted curves.

We have also investigated optically thin palladium films evaporated on different silicon-based substrates following their exposure to various concentrations of hydrogen gas in air. We used laser modulated reflectance, off the palladium surface of silicon oxide, silicon nitrite and polycrystalline silicon substrates, to recover information regarding changes in the optical properties of the samples due to the absorption of hydrogen (Fig. 9).

This resulted in both reflectivity inversion effects and the transition between $\alpha$ - and $\beta$-phases of the palladium complex after gas cycling (Fig. 10). Simple index of refraction arguments proved sufficient to explain these results [26-28]. Moreover, structural changes of the palladium films were investigated using atomic force microscopy before and after hydrogen exposure, where nanostructure formation was highlighted as a possible means of fabricating nano-devices [29].

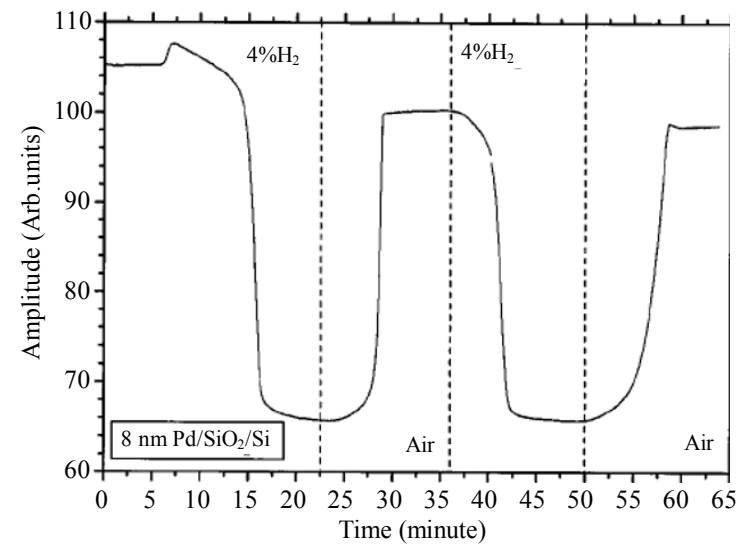

Fig. 9 Typical reflectivity changes demonstrated by an 8-nm $\mathrm{Pd}$ on $\mathrm{SiO}_{2} / \mathrm{Si}$ on hydrogen cycling.

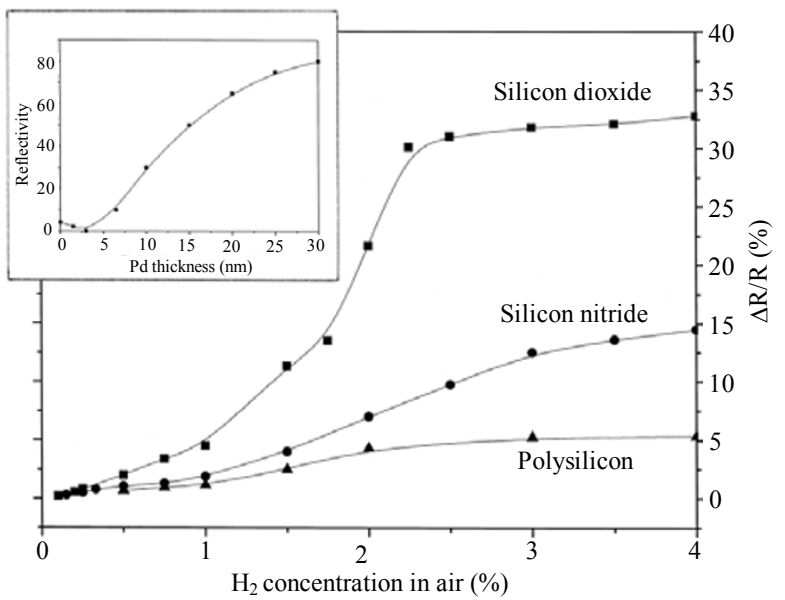

Fig. 10 Fractional reflectivity changes for various hydrogen concentrations for 8-nm palladium thin films on the different substrates (the upper left corner curve corresponds to fitted data from absolute reflectivity measurements of different film thicknesses of $\mathrm{Pd}$ on $\mathrm{SiO}_{2}$ ).

I will continue with a description of research that almost exclusively focuses on the formation and utilization of gratings in optical fibers. Far from being conventional gratings, they are based on a new grating type, on femtosecond laser inscription and gratings in polymer optical fibers. All of this work is novel and represents several firsts.

\section{Type IA Bragg gratings}

I begin this section discussing the development 
of type IA fiber Bragg gratings as sensors, whilst also seeking to explain the mechanism for the formation of this new grating type [30]. The type IA FBGs are written under longer ultraviolet (UV) exposure times than used for the inscription of more standard type I or type IIA gratings, although improvements in their inscription have shown that they can be readily inscribed in a suitably prepared optical fiber [31, 32]. The type IA may be considered a subtype of type I gratings. Their inscription characteristics however are distinct, exhibiting a large increase in the mean core index, identifiable as a large red shift in the grating Bragg wavelength $\lambda_{\mathrm{B}}$. This wavelength shift saturates and it is dependent on fiber type and hydrogenation conditions, but for a highly doped fiber (either high Ge dopant or B/Ge co-doped fiber), it is typically in the order of $15 \mathrm{~nm}$ to $20 \mathrm{~nm}$, and $5 \mathrm{~nm}$ to $8 \mathrm{~nm}$ for SMF-28 fiber. The maximum wavelength shift translates to a mean index increase of up to $2 \times 10^{-2}$. Furthermore, type IA gratings have been shown to exhibit the lowest temperature coefficient of all grating types reported to date, which makes them ideal for use in a temperature compensating, dual grating sensor [31, 33].

\section{Temperature coefficients and radiation study}

We have developed ways of tailoring the temperature and strain coefficients of fiber Bragg gratings (types I and IA) by influencing the photosensitivity pre-sensitization of the optical fiber. Controlling the level of hydrogen saturation via hot and cold hydrogenation, can produce gratings with tailored thermal and strain coefficients, resulting in a significant improvement in the matrix condition number, which impacts on the ability to recover accurate temperature and strain data (Fig. 11) [33]. The key advantages of this scheme are the utilization of two Bragg gratings having good wavelength proximity thereby avoiding costly multiplexing schemes, quick and efficient inscription using a single phase mask, common annealing cycles, and the precise placement of sensors located in a compact sensor head.

IF01001410101 (B/Ge)

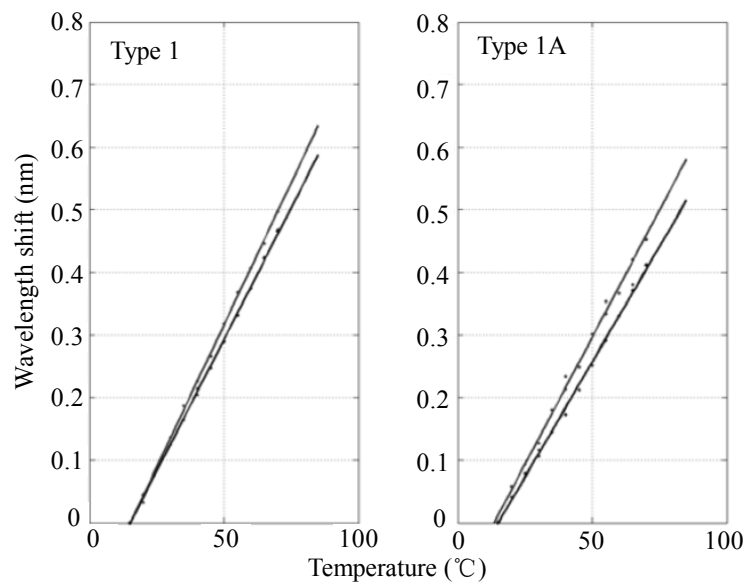

Fig. 11 Thermal coefficients for type I (left-hand traces) and type IA (right-hand traces) gratings written in $\mathrm{B} / \mathrm{Ge}$ co-doped optical fiber, for hot (grey) and cold (black) hydrogenation conditions.

Type IA gratings have lower temperature stability as compared to standard gratings; this is an indication of a higher structural damage due to the grating inscription with prolonged UV-exposure. Such a damaged structure is more sensitive to ionizing radiation as compared to the structure of type I gratings. Figure 12 shows a very large Bragg peak shift of $190 \mathrm{pm}$ for a type IA grating written in PS-1250 fiber at a radiation dose of $116 \mathrm{kGy}$ [34].

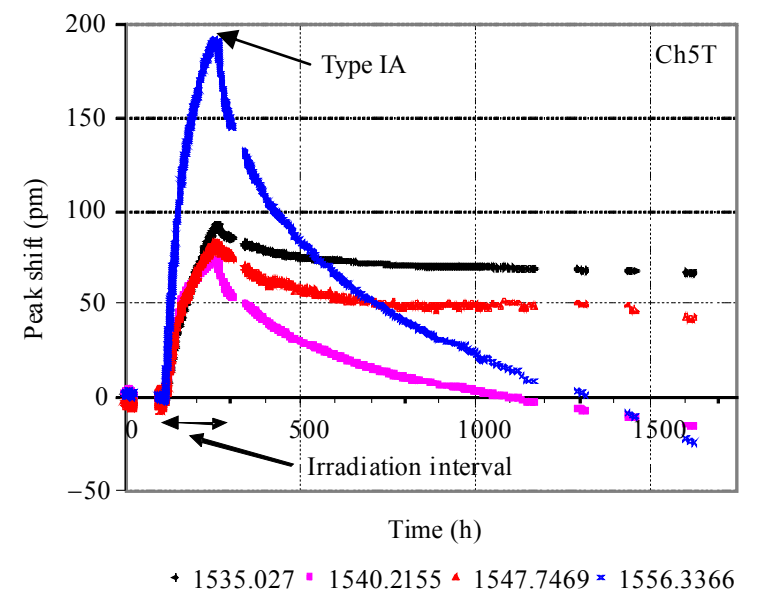

Fig. 12 Change of Bragg grating wavelength on radiation exposure (gratings at $1535 \mathrm{~nm}$ and $1548 \mathrm{~nm}$ are type I and at $1540 \mathrm{~nm}$ and $1556 \mathrm{~nm}$ are type IA).

We undertook the first experimental 
measurement on the spectral modification of type IA FBGs, instigated by moderate power near infra-red lasers [35]. The grating properties were modified in a controlled manner by absorbing guided laser light at close to $1400 \mathrm{~nm}$ that coincided with an absorption feature associated with $\mathrm{GeOH}$. This absorption band grew during the grating inscription and was due to the optical fiber preconditioning. The absorption occurred in the grating region, leading to a local increase in temperature. This wavelength selective absorption led to a reversibly modified center wavelength and chirp. A low laser power of only $10 \mathrm{~mW}$ induced small but significant wavelength shifts of approximately $100 \mathrm{pm}$. Using a more powerful source $(350 \mathrm{~mW})$ induced $\mathrm{FBG}$ wavelength shifts in excess of $750 \mathrm{pm}$ and a $30 \%$ increase in bandwidth (Fig. 13). This has serious implications for all grating types when the fiber undergoes photosensitivity preconditioning, as their spectrum can be modified using purely optical methods (no external heat source acts on the fiber), and it is also of relevance for long-term grating stability. It should be noted that high power lasers are increasingly being used in optical networks and this study may have greater implications for all grating types, as laser powers and the useable wavelength spectrum increase.

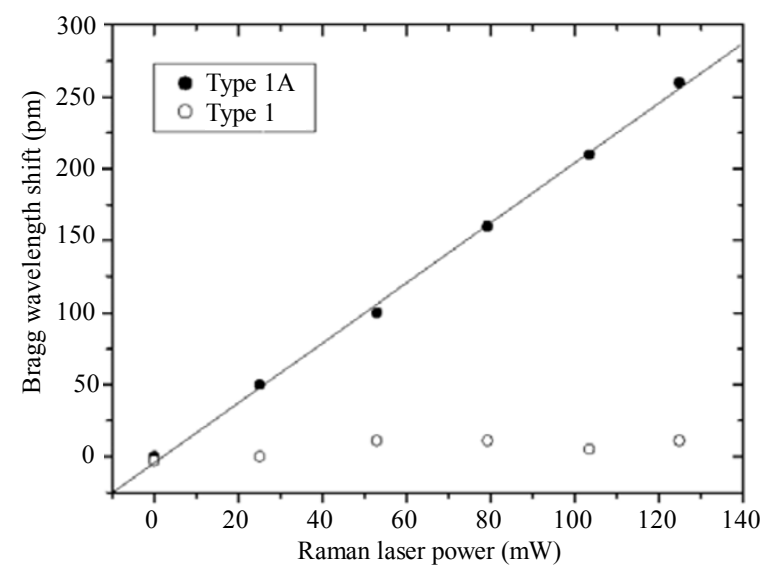

Fig. 13 Wavelength shifts of in-line types I and IA FBGs when heated with a Raman source $(1425 \mathrm{~nm}$ ) (this clearly shows a selective and controllable Bragg wavelength change for the type IA grating and none for the type I grating).

\section{Femtosecond laser micromachining and inscription in optical fibers}

An area that is particularly topical in recent years is the use of femtosecond laser systems to induce refractive index changes or ablation in transparent materials. A focused femtosecond laser pulse can fundamentally change a material's physical properties through strong non-linear absorption of the laser energy, allowing for the fabrication of intricate microstructures on the surface of opaque materials, or within the bulk volume of optically transparent glass or polymeric materials. This is widely recognized as an important development in the innovation of advanced components in fields such as medicine (stent production) and photonics (micro-devices and sensors) [36]. I will discuss the following activities: fiber Bragg, long period and superstructure grating inscription; the inscription of channels into the surface of optical fibers and it's combination with gratings as sensors. These examples show how the femtosecond laser can be used to develop different types of micro-optic devices inscribed using the same basic inscription platform and laser system, highlighting its great flexibility and potential for rapid prototyping. For example, we have shown the development of femtosecond-laser inscribed phase masks (Fig. 14) [37].

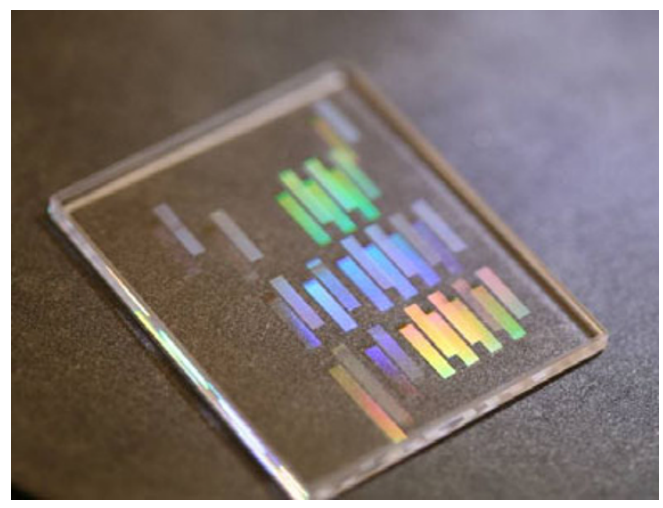

Fig. 14 Femtosecond laser inscribed phase mask below the surface of the UV grade fused silica blank.

\subsection{Bragg gratings}

The use of femtosecond lasers for the inscription 
of Bragg and long period gratings has made possible the creation of gratings with special features, for which their development would be difficult with the conventional UV laser inscription techniques. Fiber Bragg gratings have periodic refractive index modulation of the core with a sub micron pitch, reflecting light at the Bragg resonance wavelength with the forward guided mode coupling to a backward mode and to cladding modes. On the other hand, long period gratings (LPGs) have much larger period (few hundred microns) and couple light from the core to forward propagating cladding modes producing broad spectral loss bands. Many methods have been used to inscribe gratings in optical fibers, the majority of which utilize the interference of overlapping laser beams to create the desired fringe pattern and spacing. An alternative approach is step-wise inscription whereby single laser pulses are used to induce a refractive index change corresponding to an individual grating plane, with the grating inscribed one step at a time along the fiber core. With UV laser inscription there is a relatively long processing time to form the grating and so possible errors in the grating spacing due to thermal effects and/or small variations in the fiber's strain can occur, limiting the gratings to a very short length [11-13]. Similar point-by-point inscription can be realized using a femtosecond laser [38], but with the advantage that the laser fires a rapid train of precisely timed pulses with good energy stability; the translation stage moves with constant speed in a rapid process that takes only a few seconds. The main advantage of the femtosecond writing approach lies in its flexibility to alter the Bragg grating parameters, allowing for variations in the grating length, pitch, and spectral response to be incorporated easily. The Bragg peak wavelength can be externally tailored, by tuning the ratio of the translation speed to the laser pulse repetition rate. Moreover, the nature of the induced material changes can be used for inscribing gratings in almost every waveguide material, not just silica, as long as there is a transparent access to the waveguide. The physical inscription mechanism of gratings with infrared femtosecond pulses is nonlinear absorption. The multi-photon process requires power densities (pulse energies) that are just above a material-dependent threshold. As a result, there exists an optimal pulse power for every focusing condition, demanding precise control of the pulse energy. However, this nonlinear process makes it possible to overcome the diffraction limit and to inscribe fine periodic structures that would not be feasible with linear absorption processes.

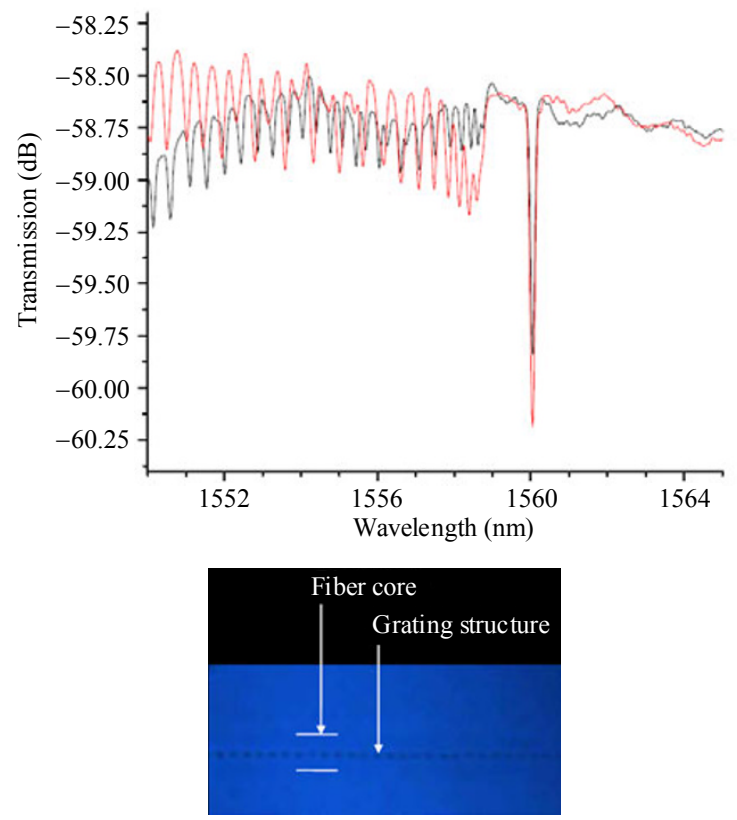

Fig. 15 Spectra for first and second order femtosecond laser inscribed FBG (top), FBG position in the fiber core (bottom).

It is also possible to inscribe first order Bragg gratings in step-index fiber with a relatively low numerical aperture (NA) lens and without the use of oil immersion techniques (Fig. 15) and second order FBGs in single-mode, microstructure optical fiber (Fig. 16), both using a near infrared femtosecond laser (HighQ Laser Femtoregen IC 355), via the point-by-point method [38]. With a suitable fiber design the laser pulses penetrate the holes of the microstructure fiber without significant pulse break-up during inscription, and without using techniques that "remove" the holey structure by 
filling with index matching oils. This is an important, generally applicable step in using femtosecond lasers for FBG inscription in optical fibers.

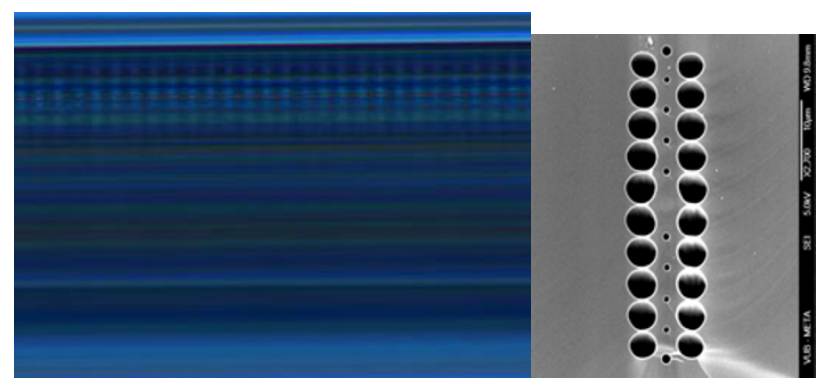

Fig. 16 FBG in microstructure fiber (left) and cross section of highly birefringent microstructure fiber (right).

The inscribed FBG in the microstructure optical fiber has wavelength to strain responsivity and temperature responsivity of $0.965 \mathrm{pm} / \mu \varepsilon$ and 11.3 $\mathrm{pm} /{ }^{\circ} \mathrm{C}$, respectively (Fig. 17). These are very similar to the results for conventional FBGs.

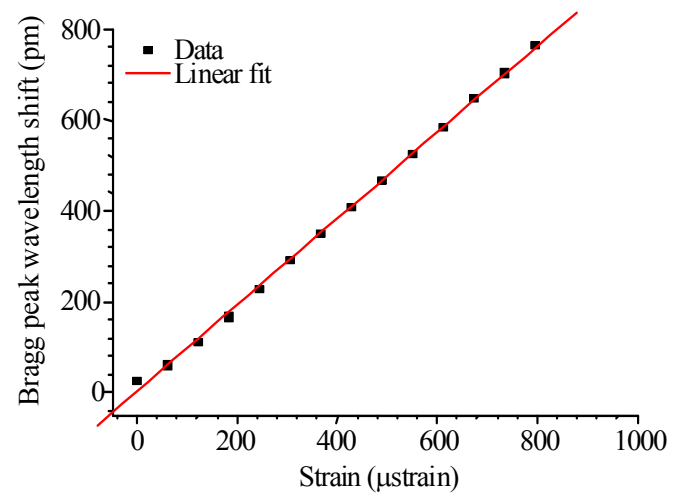

Fig. 17 Typical strain response of femtosecond laser inscribed FBG.

There are two key drawbacks of femtosecond inscribed gratings: (1) high levels of broad spectrum losses, incurred during inscription [39] and (2) the significant degree of polarization dependence of grating devices [40]. These effects can be problematic for filter applications in optical communications and sensing. The complexity of multi-channel optical networks has made them more sensitive to polarization dependent losses and associated wavelength shifts. Moreover, the multiplexing of optical fiber sensors demands low loss devices with minimal polarization dependent characteristics for accurate wavelength encoded demodulation of signals. We have shown that it is possible to inscribe Bragg gratings using a femtosecond laser having an insertion loss of less than $0.4 \mathrm{~dB}$ over a grating length of $20 \mathrm{~mm}$, with minor polarization dependence, measured as less than $5 \mathrm{pm}$ with a polarization induced transmission loss of less than $0.1 \mathrm{~dB}$ (Fig. 18). Reasonable grating strengths of up to $3 \mathrm{~dB}$ were recorded [41].
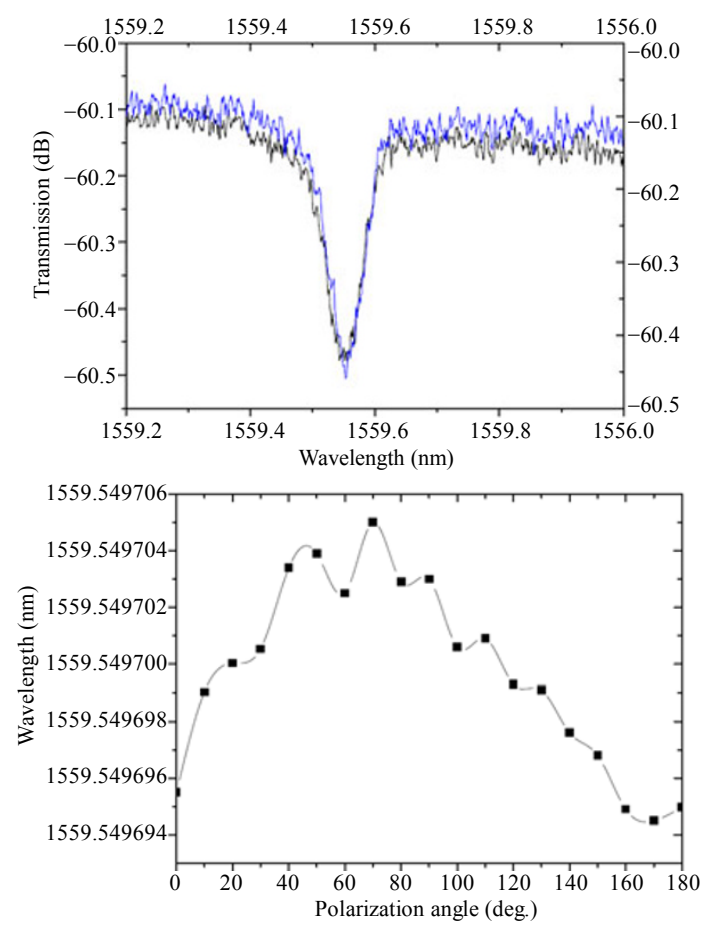

Fig. 18 Transmission spectra for two orthogonal polarization states of a femtosecond laser inscribed FBG (top) and wavelength change with polarization angle of less than $5 \mathrm{pm}$ (bottom).

\subsection{Long period gratings}

The approach discussed above may be adapted for use with long period grating inscription. UV laser inscription of LPGs produces a uniform periodic index perturbation throughout the core of the photosensitive fiber. In coupled mode theory, the light travelling through the fiber core is assumed to be linearly polarized thus simplifying the calculations, whilst maintaining the key results. Bound by this approximation, as there is no azimuthal dependence within the fiber core, the UV LPG will only couple to cladding modes of 
azimuthal order 1 [42]. As the femtosecond inscribed gratings have a far smaller focal point, with a local index change in a small section of the fiber core, there is now angular and polarization dependence on the femtosecond laser inscribed gratings (much as for small and large angle tilted gratings). Mathematically, the coupling efficiency between two modes has an extra term, coupling between all azimuthal modes becomes possible:

$$
\int_{0}^{2 \pi} e^{j(l-1) \phi} d \phi=2 \pi \delta_{l 1} .
$$

As a result, the confinement of femtosecond inscribed gratings and the localized refractive index (RI) change associated with this confinement has two different properties compared to the uniform RI change throughout the core of UV laser inscribed gratings. Firstly, a polarization dependency arises as different polarizations of light will see the RI perturbation differently. Secondly, the azimuthal dependence now means that the cladding mode coupling is not limited to $L P_{1 l}$ but can now couple to multiple sets of cladding modes $L P_{m l}$. For sensing, this opens up the possibility of coupling to more sensitive cladding modes than are possible compared with standard UV inscribed grating devices. Typical transmission spectra for a LPG in air and immersed in a RI liquid with a surrounding refractive index (SRI) of 1.444 are shown in Figs. 19(a) and 19(b), respectively. In air there are two strong and three weak attenuation peaks, whereas when the LPG is immersed in RI liquid with an SRI of 1.444 , two strong peaks remain with two weak ones. This suggests that the effective indices of the cladding modes associated with the peaks are higher than 1.444, and that coupling to lower order modes is occurring. If the LPG is UV inscribed the attenuation peaks will all experience the same wavelength shift with increasing surrounding refractive index. However, this is not the case for femtosecond LPGs as they couple to multiple sets of cladding modes simultaneously, and this behavior gives rise to multiple attenuation peaks with both blue and red wavelength shifts with increasing surrounding refractive index. By using the combined shift of two opposing attenuation peaks a greater RI sensitivity can be achieved (Fig. 20). We have measured a maximum RI sensitivity of 1680 $\mathrm{nm} / \mathrm{RIU}$.

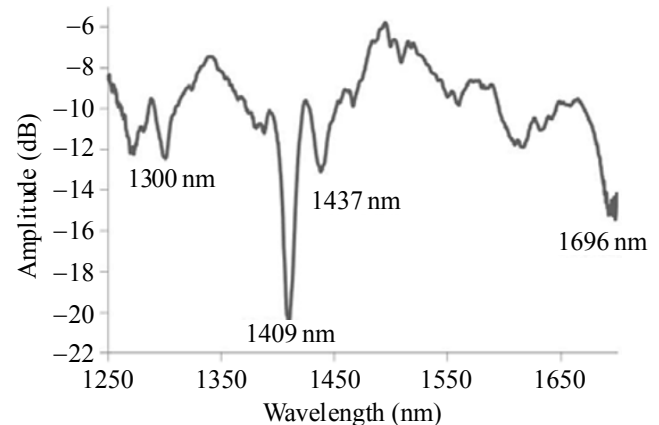

(a)

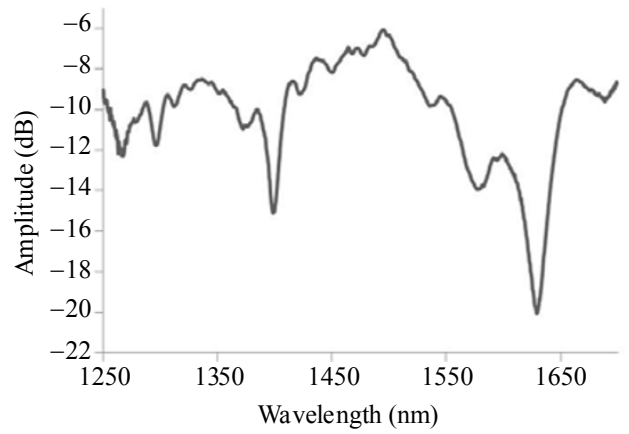

(b)

Fig. 19 The transmission spectra of the femtosecond induced LPG of period $400 \mu \mathrm{m}$ in (a) air and (b) a liquid of SRI of 1.444

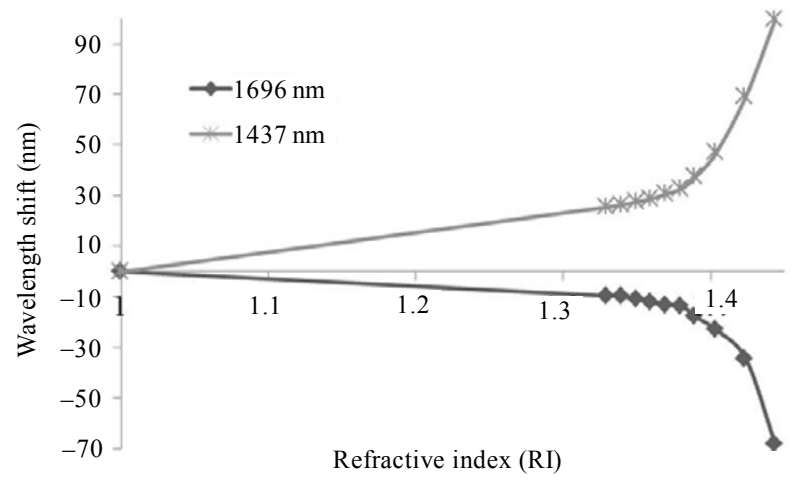

Fig. 20 Peak refractive index sensitivity of the LPG and the numerically calculated values.

\subsection{Superstructure gratings}

The superstructure fiber grating ( $\mathrm{SFG}$ ) is a composite grating structure formed when the Bragg 
grating is exposed to a slowly varying refractive index (amplitude) modulation along the grating length thereby inducing counter-directional mode coupling, resulting in a comb filter response viewed in reflection [43, 44]. There are numerous applications demonstrated for the SFG such as tuneable distributed Bragg reflectors for fiber lasers and multi-channel dispersion compensators.

One key advantage of the femtosecond point-by-point inscription approach is that the impact of co-directional cladding mode coupling affecting the Bragg grating reflection response can be effectively eliminated during inscription. This is possible because the femtosecond inscription leads to both positive and negative index changes, resulting in a spatially averaged index change that is more than ten times less than that for UV inscribed devices. The gratings can be written with well defined reflectivities and at design wavelengths that precisely coincide with the desired Bragg wavelength. Finally, the femtosecond gratings are of very low loss, displaying uniform spectra, which are deemed extremely important where it is necessary for the light to penetrate the full grating length and for each of the individual grating elements to contribute to the reflection response, e.g. within the Fourier design limit, which demands that gratings have a reflectivity of less than $25 \%$.

Figure 21(a) shows a transmission spectrum for an LPG accompanied by one for an SFG, and both devices have an amplitude modulation period of 600 microns, but in the latter case the laser energy is reduced by $5 \%$, which proves sufficient to completely suppress the LPG response. However the laser pulse energy remains just above the material-dependent threshold to allow for the SFG. Figure 21 (b) shows transmission and reflection spectra for an SFG having a Bragg period of $1078 \mathrm{~nm}$, while the amplitude period is 300 microns. There is no evidence of the LPG response, but we observe the characteristic Bragg cladding mode resonances.

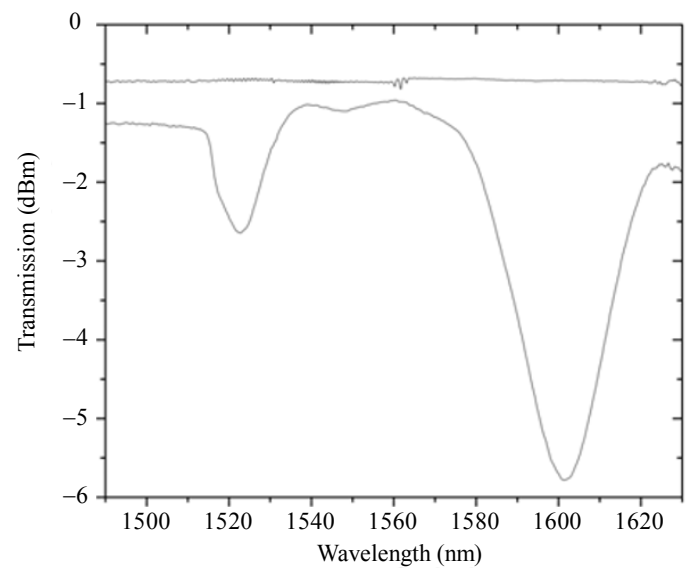

(a)

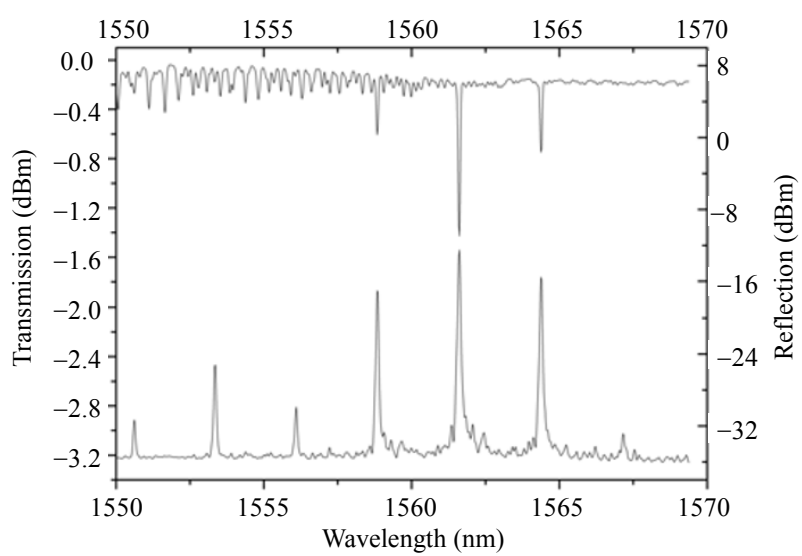

(b)

Fig. 21 (a) Transmission spectrum for LPG and SFG (with LPG completely suppressed) for amplitude modulation period of $600 \mu \mathrm{m}$, (b) SFG transmission and reflection response for a Bragg period of $1078 \mathrm{~nm}$, and amplitude period of $300 \mu \mathrm{m}$.

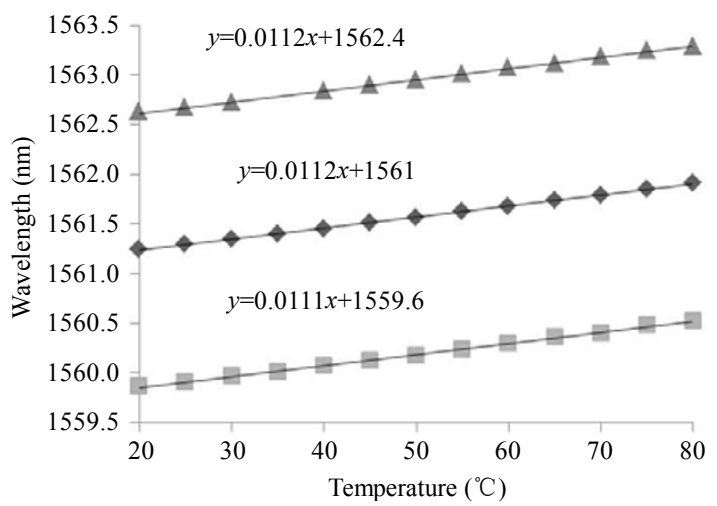

Fig. 22 Measured temperature response of the three strongest reflection peaks of a typical SFG device. 
In Fig. 22 we observe the measured temperature response of the three strongest reflection peaks of a typical femtosecond laser SFG device, producing a wavelength response to temperature that is typical for a $\mathrm{FBG}$ based device of $11.2 \mathrm{pm} /{ }^{\circ} \mathrm{C}$, for each peak.

\section{Modeling femtosecond laser inscribed fiber gratings}

We have seen how the highly localized refractive index changes, confined to the laser beam focal volume, permitting the development of complex gratings, such as sampled gratings. On the other hand this localized index change can be a drawback, as a small lateral shift of the focal point will displace the inscribed grating from fiber axis (off-axis inscription), changing the grating spectrum in strength and shape, because of symmetry breaking. It is important to predict how it will modify the FBG spectrum, and numerical modelling can become a strong tool to support the inscription process. To date there are no noteworthy studies of the grating spectra of femtosecond laser inscribed gratings by the point-by-point method. We have applied the bidirectional beam propagation method (BiBPM) and the finite element method (FEM), optimizing the solution convergence and accuracy for the propagating mode calculation. BiBPM is well suited to modeling coupled forward and backward waves, readily accounting for resonant effects of gratings $[45,46]$. Our analysis can predict losses and gives prominence to the cladding mode spectra (Fig. 23). We have shown that a lateral shift of the grating leads to an exponential reduction in FBG reflectivity, a concomitant modification of the cladding modes, and the growth of a ghost mode (Fig. 24).

For lateral displacements greater than $1 \mu \mathrm{m}$, the gradient of the slope increases rapidly leading to lower reflectivity even for small displacements; in agreement with experimental observations (Fig. 25).

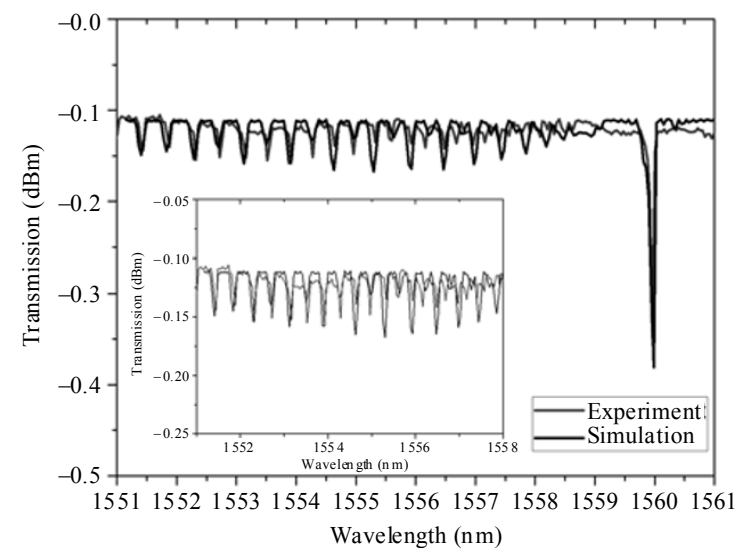

Fig. 23 Experimentally measured and simulated spectra showing excellent agreement in the number of cladding modes and their wavelength positions (the simulation resolution is set to be equal with experimental measurements resolution).

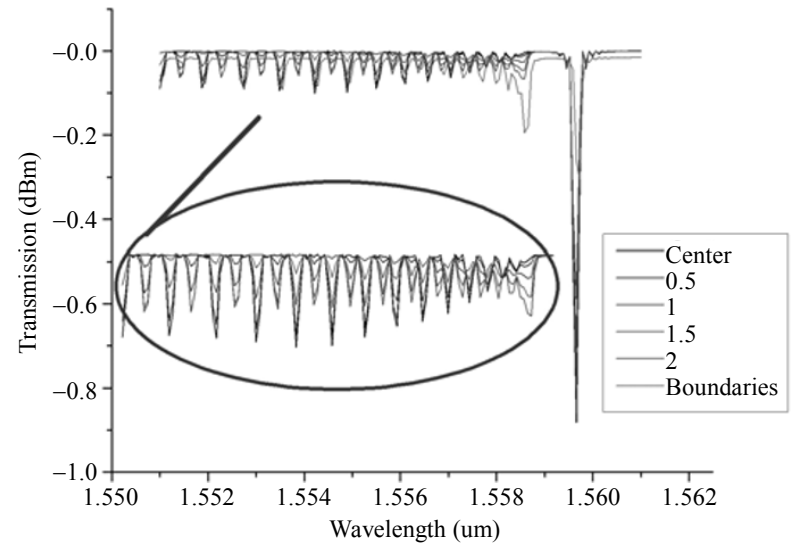

Fig. 24 Grating spectra for different offsets from the center of the core, where the label refers to offset in microns (inset: cladding mode structure with greater clarity).

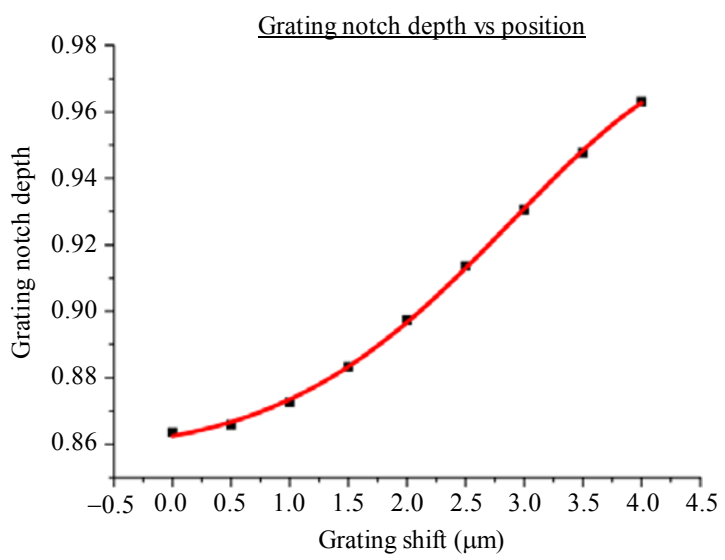

Fig. 25 Grating notch depth proportional to the position of the grating in the fiber core (line is a Gaussian fit). 


\section{Long period gratings in photonic crystal fiber-electric arc and femtosecond laser inscription}

The properties of LPGs fabricated in photonic crystal fiber (PCF) were investigated. We have used both the electric arc and the femtosecond point-by-point methods for LPG inscription, and have extracted some significant results, such as the temperature insensitivity of the LPG, it's applicability to bend sensing and to refractive index sensitivity. The bend sensitivity resulted from two different sources, firstly an asymmetry in the holey fiber cross-sectional geometry and secondly asymmetry induced when using a femtosecond laser for inscription of the grating.

\subsection{Femtosecond laser LPGs as bend sensors}

A series of symmetric and asymmetric LPGs were inscribed in PCF using a femtosecond laser system. We have found that asymmetric (off-center) femtosecond laser inscription of LPGs produces devices with certain attenuation bands that have a spectral sensitivity to the orientation of the curvature. Spectral curvature sensitivities of $-11.4 \mathrm{~nm} \cdot \mathrm{m}$ for a concave bend and $+7.0 \mathrm{~nm} \cdot \mathrm{m}$ for a convex bend were measured. The great advantage with PCF is the low temperature cross-sensitivity [47]. All the fabricated LPGs exhibit spectral birefringence ranging from $1 \mathrm{~nm}$ to $10 \mathrm{~nm}$ and this is related to the inscription method and the laser energy used to fabricate the LPG. The symmetrically inscribed LPGs exhibit no dependence on bend sensitivity with fiber orientation. The transmission spectra of an asymmetric LPG are shown in Fig. 26.

\subsection{Electric arc LPGs as bend sensors}

In the case of LPGs inscribed using a spatially-periodic electric arc, the whole fiber cross section is affected uniformly and so one expects a sensor response that is not vectorial in nature. However, strong bend sensitivity can exist if the fiber cross section has inherent asymmetry (Fig. 27).

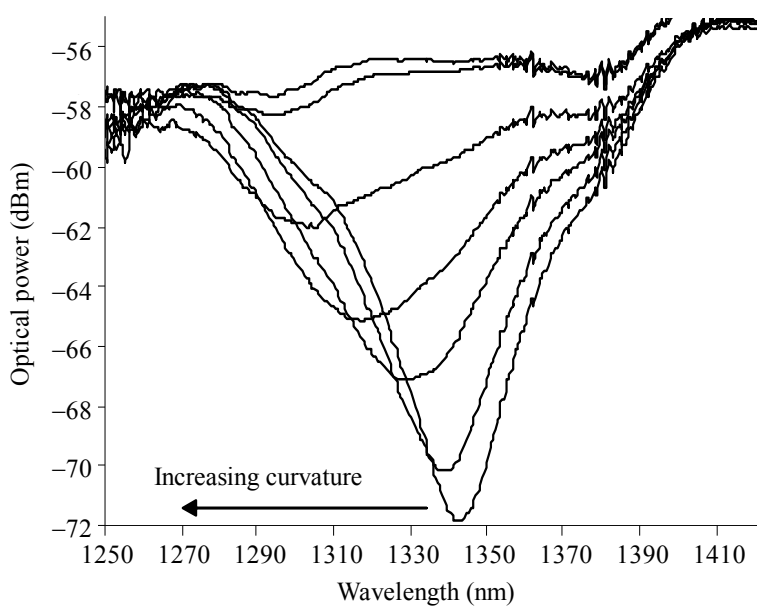

(a)

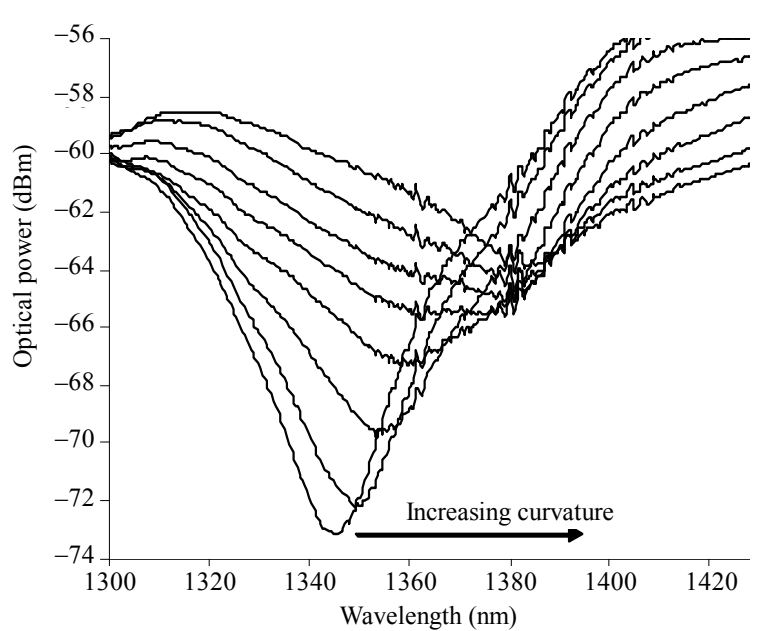

(b)

Fig. 26 Curvature response transmission spectra for an asymmetrically inscribed LPG written in PCF (period $=400 \mu \mathrm{m}$, inscription energy $=410 \mathrm{~nJ})$ (LPG is subjected to (a) concave and (b) convex bending).

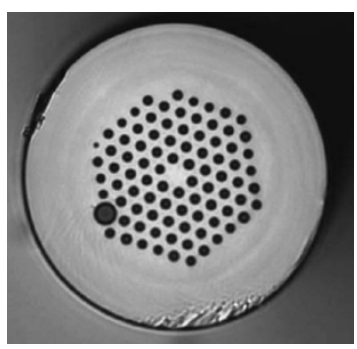

Fig. 27 PCF cross-section of endlessly single mode fiber with inherent asymmetry.

LPGs in two different types of PCF have been investigated for their sensitivity to temperature, bending, surrounding refractive index, and strain [48-52]. Both fibers show negligible temperature 
sensitivity $\left(<6 \mathrm{pm} /{ }^{\circ} \mathrm{C}\right)$ whilst having significant sensitivity to other measurands. This is an important factor, since one of the persistent problems of using LPGs as sensors is their cross-sensitivity to temperature, which results in discriminatory schemes needing to be employed to separate the effect of temperature from the desired measurands [49]. The two fibers have different responses to RI changes, with the asymmetric fiber design showing a useful response, whereas the symmetric endlessly single mode PCF displays minimal changes to SRI, which would make it better suited to applications involving sensor embedding. The directional bend sensitivity of the LPG in the asymmetric cross-section PCF was $d \lambda / d R=(-12.4 \pm 1.2) \mathrm{nm}$ and $\mathrm{d} \lambda / \mathrm{d} R=(9.64 \pm 1.0) \mathrm{nm}$ for bending in opposite directions (Fig. 28). This directional bend sensitivity, which is attributed to the disrupted symmetry of the photonic crystal, compares well with other reported sensors and is the first time that this behavior has been seen in LPGs fabricated in PCF.

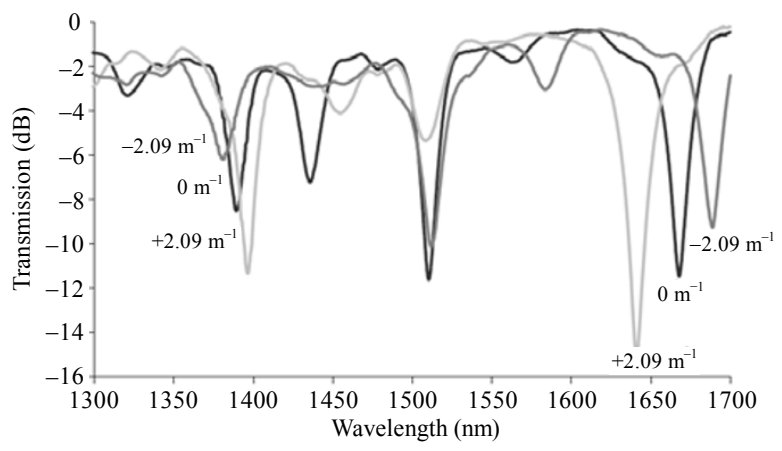

Fig. 28 Spectral response of the asymmetric cross-section PCF to bending (black line: LPG with no curvature; dark grey line: curvature of $-2.09 \mathrm{~m}^{-1}$ in the $180^{\circ}$ position; light grey line: curvature of $+2.09 \mathrm{~m}^{-1}$ in the $0^{\circ}$ position).

These experiments show the potential that LPGs fabricated in PCF have as sensing devices and suggest that PCF could in principle be designed for optimum sensitivity to desired measurand(s), whilst minimizing or removing undesirable cross sensitivity.

Perhaps two pieces of work have the particular significance, where we have developed composite devices to realize firstly, a fiber optic magnetic sensor using the broad capabilities of a single femtosecond laser inscription platform and secondly, a tunable grating filter in polymer optical fiber.

\section{Magnetic field sensor}

Begining with the direct current (DC) magnetic field sensor, it was specifically designed for the detection and characterization of static magnetic fields. Magnetic sensors have been widely used to control and analyze a wide range of devices such as linear and rotary position sensors and current sensors. Optical detection methods that use interferometery [53], Faraday rotation and cantilever arrangements, covering fields from $1 \mathrm{nT}$ to $1 \mathrm{~T}$, have been used [54-55]. Most reported studies were conducted using high frequency magnetic fields or using mumetal shields for detecting either slowly varying or static magnetic fields, leading to practical application issues. Therefore, magnetic sensors would greatly benefit from a sensitive optical detection method for which static magnetic fields could be measured and with sensor information that is encoded as an absolute quantity, such as the wavelength encoding offered by a fiber Bragg grating sensor.

By carefully tailoring the inscription threshold for the index change and ablation, we incorporated a fiber Bragg grating and a micro-machined rectangular slot into the same optical fiber on a single inscription platform. The symmetry of the fiber was broken by the micro-slot, producing non-uniform strain across the fiber cross section, whilst also offering the potential for vectorial sensing (Fig. 29). The sensing region was then coated with Terfenol-D, which in the presence of magnetic fields changed dimensions through magnetostriction, making the device sensitive to static magnetic fields. The advantage of the Bragg grating is a sensor output that is directly wavelength 
encoded, leading to simple demodulation by monitoring wavelength shifts that occurs as the fiber structure changes shape in response to the external magnetic field [56].

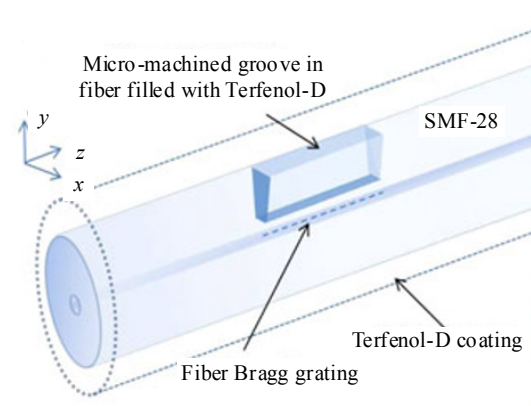

(a)

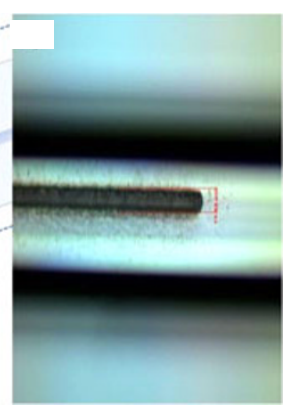

(b)
Fig. 29 (a) Schematic of the magnetic optical fiber sensor geometry, with femtosecond laser inscribed FBG in the core and micro-slot, all coated with Terfenol-D and (b) microscope image of the slot in the Corning SMF-28 prior to being filled and coated.

The Terfenol-D when sputtered onto the optical fiber fills the micro-slot creating a robust, monolithic structure. The resulting sensors demonstrate high sensitivity to weak static magnetic field and sensitivity to the magnetic field direction, which has previously not been demonstrated with comparable approaches. It has demonstrated a minimum detection limit of $0.047 \mathrm{mT}$ and a sensitivity of $0.3 \mathrm{pmmT}^{-1}$ in transmission with a resolution limit of $\pm 0.3 \mathrm{mT}$ in a magnetic field strength of approximately $20 \mathrm{mT}$ (Fig. 30).

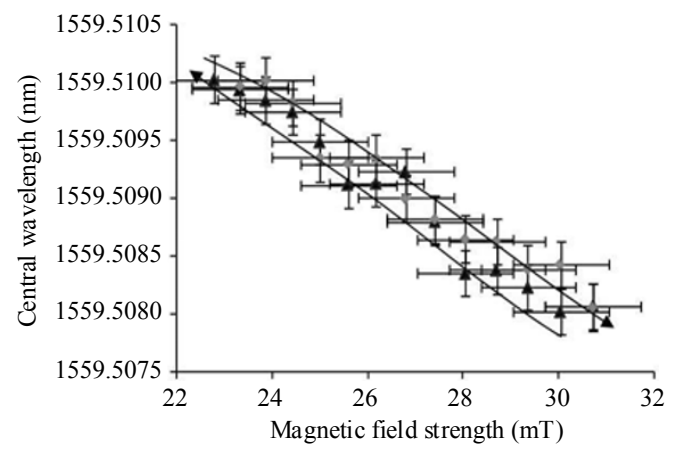

Fig. 30 Spectral characteristics of the device in transmission as a function of magnetic field strength.

The simplicity of the optical configuration and results show the potential for this technique to be applied to a range of fields due to its small sensor size and ability to be remotely interrogated. This demonstrates the potential for vectorial magnetic field sensing with high sensitivity and large scope for flexibility.

\section{Polymer optical fiber gratings}

In recent years, my research has been founded on the development of fiber Bragg grating technology in polymer optical fibers. A significant outcome is the development of the first tunable grating filter in polymer optical fiber (POF), which will be discussed briefly in this paper [57, 58]. However, we have realized several other firsts, such as Bragg grating inscription in microstructure polymer optical fiber, in novel polymers such as TOPAS ${ }^{\circledR}$ (a cyclic olefin copolymer that has the potential for label-free bio-sensing when combined with Bragg gratings), and at wavelengths that are the shortest reported to date, operating at $827 \mathrm{~nm}$, (Fig. $31)[59-62]$ and as bend sensors $[63,64]$.

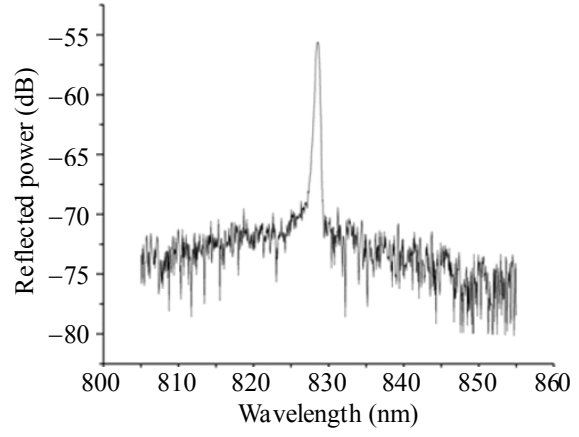

Fig. 31 Reflection spectrum of an FBG fabricated in multi-mode microstructure polymer optical fiber at $827 \mathrm{~nm}$ with bandwidth resolution of $0.5 \mathrm{~nm}$.

We have exploited the high temperature sensitivity of FBG in polymer optical fiber to realize an electrically tuneable filter. A grating recorded in a polymethyl methacrylate (PMMA) based step-index fiber was coated with a $\mathrm{Pd} / \mathrm{Cu}$ metallic layer: the former to aid adhesion to the PMMA and the latter to act as a thin film heating element. Because of the risk of grating erasure near the glass transition temperature, the fiber was coated at room 
temperature using 172-nm radiation, thereby undergoing vacuum decomposition of a metal-organic film whilst at the same time causing chemical roughening of the POF device to aid adhesion.

Figure 32 shows the electrical tuning characteristic of the device, which demonstrates a sensitivity of $-13.4 \mathrm{pm} / \mathrm{mW}$, and the time constant is $1.7 \mathrm{~s}^{-1}$.

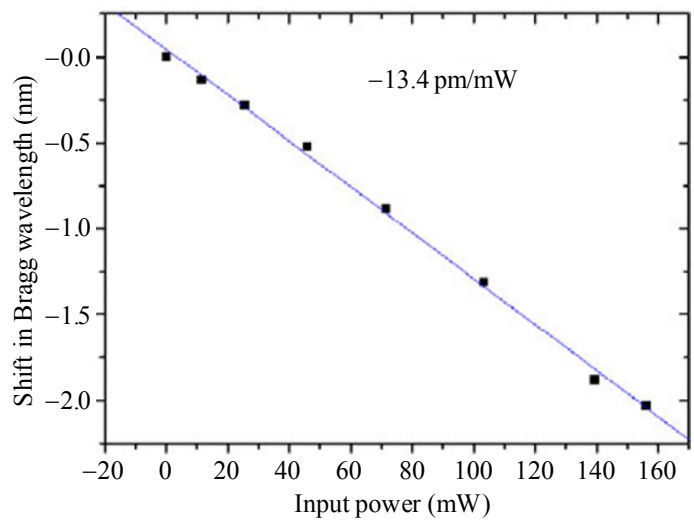

Fig. 32 Wavelength shift induced by ohmic heating of the polymer optical fiber grating.

When the input power is uniform the temperature variation as a function of time is

$$
T(t)=\frac{P_{\text {in }}^{\prime}}{a}[1-\exp (-a t)]
$$

where $P_{\text {in }}^{\prime}$ is related to the input power, but depends on the thermal capacity of the PMMA and the metal film. A measurement of $a$ yields information regarding the rate of heat flow out of the fiber.

\section{Other work}

The sections above describe certain areas of my research during the period 1996 to 2010; other activities include the development of surface plasmon sensors [65-67], the use of femtosecond lasers to modify the sensing characteristics of optical fiber sensors and the development of microfluidic devices.

\section{Acknowledgement}

I would like to acknowledge several outstanding scientists that I have worked closely with for many years. Of particular note are David Webb, Tom Allsop, Andreas Othonos, and Mike Komodromos. We have shared success, failure, and many long hours in the lab. I would also like to thank the many students over the years, for their collaboration, patience, and resilience; seeing them mature as scientists has been superb. Finally, my thanks go to Professor David Jackson for nurturing my interest in the optical sciences, for always finding time for a chat and for openly sharing his vast knowledge.

Open Access This article is distributed under the terms of the Creative Commons Attribution License which permits any use, distribution, and reproduction in any medium, provided the original author(s) and source are credited.

\section{References}

[1] K. Kalli, "Investigation of linear and non-linear optical phenomena using high finesse ring resonators," Ph.D. dissertation, Physics Department, the University of Kent, UK, 1992.

[2] K. Kalli, D. Culverhouse, and D. A. Jackson, "Fiber frequency shifter based upon stimulated Brillouin scattering generation in high finesse ring resonators," Opt. Lett., vol. 16, no. 19, pp. 1538-1540, 1991.

[3] D. Culverhouse, K. Kalli, and D. A. Jackson, "Stimulated Brillouin scattering ring resonator laser for SBS gain studies and microwave generation," Elec. Lett., vol. 27, no 22, pp. 2033-2035, 1991.

[4] K. Kalli and D. A. Jackson, "Investigation and applications of all-fiber Brillouin ring resonator lasers," Fiber \& Int. Opt., vol. 14, no. 4, pp. 303-330, 1995.

[5] K. Kalli and D. A. Jackson, "Ring resonator optical spectrum analyzer with $20 \mathrm{kHz}$ resolution," Opt. Lett., vol. 17, no. 15, pp. 1090-1092, 1992.

[6] K. Kalli, M. Berwick, and D. A. Jackson, "Recent developments of fiber optic components for LDV signals," in Laser Techniques and Applications in Fluid Mechanics: Proceedings of the 6th International Symposium, Lisbon, Portugal, July 20-23, pp. 25-43, 1992.

[7] K. Kalli and D. A. Jackson, "Tunable fiber frequency shifter that uses an all-fiber ring resonator," Opt. Lett., vol. 17 , no. 17 , pp. 1243-1245, 1992.

[8] K. Kalli and D. A. Jackson, "Analysis of the dynamic response of a ring resonator to a time varying input 
signal," Opt. Lett., vol. 18, no. 6, pp. 465-467, 1993.

[9] K. Kalli and D. A. Jackson, "Dynamic response of high resolution ring resonator optical spectrometers to time varying input signals," Fiber \& Int. Opt., vol. 14, no. 3, pp. 211-223, 1995.

[10] F. Farahi, K. Kalli, and D. A. Jackson, “An all-fiber ring resonator gyroscope using low coherence length source," presented at Optical Fiber Sensors Conference, OFS-6’ 89, Paris, Sept., 1989.

[11] A. Othonos and K. Kalli, Fiber Bragg gratings: fundamentals and applications in telecommunications and sensing. London: Artech House Inc., 1999.

[12] Andreas Othonos, Kyriacos Kalli, David Pureur, and Alain Mugnier, "Fiber Bragg gratings" in Wavelength Filters for Fiber Optics, vol. 123. Herbert Venghaus Ed. Heidelberg: Springer (Springer Series in Optical Sciences), 2006.

[13] A. Othonos and K. Kalli, "Bragg gratings in optical fibers," in Handbook of Advanced Electronic and Photonic Materials and Devices, vol. 9, Chapter 9 (Nonlinear Optical Materials), H. S. Nalwa Ed. San Diego: Academic Press, 2000.

[14] G. P. Brady, K. Kalli, D. J. Webb, L. Zhang, I. Bennion, and D. A. Jackson, "Extended range, coherence tuned dual wavelength interferometry using a superfluorescent fiber source and chirped fiber Bragg gratings," Opt. Comm., vol. 134, no. 1-6, pp. 341-348, 1997.

[15] G. P. Brady, K. Kalli, D. J. Webb, L. Reekie, J. L. Archambault, and D. A. Jackson, "Simultaneous interrogation of interferometric and Bragg grating sensors," Opt. Lett., vol. 20, no. 11, pp. 1340-1342, 1995.

[16] K. Kalli, G. P. Brady, D. J. Webb, L. Zhang, I. Bennion, and D. A. Jackson, "Wavelength division and spatial multiplexing using tandem interferometers for Bragg grating sensor networks," Opt. Lett., vol. 20, no. 24, pp. 2544-2546, 1995.

[17] Y. J. Rao, K. Kalli, G. P. Brady, D. J. Webb, L. Zhang, I. Bennion, and D. A. Jackson, "Spatiallymultiplexed fiber-optic Bragg grating strain and temperature sensor system based on interferometric wavelength-shift detection," Elec. Lett., vol. 31, no. 12, pp. 1009-1010, 1995.

[18] G. P. Brady, K. Kalli, D. J. Webb, L. Reekie, J. L. Archambault, and D. A. Jackson, "Simultaneous measurement of strain and temperature using the first- and second-order diffraction wavelengths of Bragg gratings," IEE Proc. in Optoelec., vol 144, no. 3, pp. 156-161, 1997.

[19] K. Kalli and D. A. Jackson, “A feasibility study into the use of Raman and fluorescence spectroscopy for pollution monitoring of volatile organic compounds in ground water," Submitted to Dr. Charles Shelton, Environmental Research Dept. Shell Research Ltd., 1996.
[20] D. Sun, K. Kalli, R. May, and R. Claus, "Optical fiber devices, sensors and systems", Final report submitted to Henry Whitesel, Code 2753, David Taylor Research Center, February, 1993.

[21] T. Kuzniz, D. Halot, A. G. Mignani, L. Ciaccheri, K. Kalli, M. Tur, A. Othonos, C. Christofides, and D. A. Jackson, "Instrumentation for the monitoring of toxic pollutants in water resources by means of neural network analysis of absorption and fluorescence spectra," Sens. \& Act. B, vol. 121, no. 3, pp. 231-237, 2007.

[22] K. Kalli, A. Othonos, and C. Christofides, "Hydrogen gas detection via photothermal deflection measurements," Rev. Sci. Inst., vol. 68, no. 9, pp. 3544-3552, 1997.

[23] K. Kalli, A. Othonos, C. Christofides, A. Spetz, and I. Lundström, "Photomodulated thermoreflectance detection of hydrogen gas using optically thin palladium film on silicon oxide," Rev. Sci. Inst., vol. 69, no. 3, pp. 1505-1511, 1998.

[24] C. Christofides, A. Othonos, and K. Kalli, "Non destructive photothermal radiometric measurements of defects and metallic contaminating impurities on silicon wafers", in Crystalline Defects and Contamination III, Electrochemical Society Proc., vol. 2001-29, pp. 153-165, 2001.

[25] K. Kalli, A. Othonos, C. Christofides, and F. Tardif, "Non-destructive evaluation of metal contaminated silicon wafers using radiometric measurements," $J$. Appl.Phys., vol. 86, no. 6, pp. 3064-3067, 1999.

[26] K. Kalli, A. Othonos, and C. Christofides, "Characterization of reflectivity inversion, $\alpha$ and $\beta$ phase transitions and nanostructure formation in hydrogen activated thin $\mathrm{Pd}$ films on silicon based substrates," J. Appl. Phys., vol. 91, no. 6, pp. 3829-3840, 2002.

[27] C. Christofides, K. Kalli, and A. Othonos, "Optical response of thin supported palladium films to hydrogen: non-destructive testing for hydrogen detection," Plat. Metals Rev., vol. 43, no. 4, pp. 155-157, 1999.

[28] K. Kalli, A. Othonos, C. Christofides, A. Spetz, and I. Lundstrőm, "Temperature-induced reflectivity changes and activation of hydrogen sensitive optically thin palladium metal films on silicon oxide," Rev. Sci. Inst., vol. 69, no. 9, pp. 3331-3338, 1998.

[29] A. Othonos, K. Kalli, and D. P. Tsai, "Optically thin palladium films on silicon based substrates and nanostructure formation: effects of hydrogen," Appl. Surf. Sci., vol. 161, no. 1-2, pp. 54-56, 2000.

[30] A. G. Simpson, K. Kalli, K. Zhou, L. Zhang, and I. Bennion, "Formation of type IA fiber Bragg gratings in germanosilicate optical fiber," Electron. Lett., vol. 40, no. 3, pp. 127-133, 2004.

[31] A. G. Simpson, K. Kalli, K. Zhou, L. Zhang, and I. Bennion, "An idealised method for the fabrication 
of temperature invariant IA-I strain sensors," presented at Optical Fibre Sensors Conference OFS16, Nara, Japan, 2003.

[32] A. G. Simpson, K. Kalli, K. Zhou, L. Zhang, and I. Bennion, "Blank beam fabrication of regenerated type IA gratings," Meas. Sci. Technol., vol. 15, no. 8, pp. 1665-1669, 2004.

[33] K. Kalli, G. Simpson, K. Zhou, L. Zhang, and I. Bennion, "Tailoring the temperature and strain coefficients of type I and type IA dual grating sensors - the impact of hydrogenation conditions," Meas. Sci. Technol., vol. 17, no. 5, pp. 949-954, 2006.

[34] A. Faustov, P. Saffari, C.Koutsides, A. Gusarov, M. Wuilpart, P. Mégret, K. Kalli, and L. Zhang, "Highly radiation sensitive type IA FBGs for dosimetry applications," presented at European Conference on Radiation Effects on Component and Systems, Sevilla, Spain, September 19-23, 2011.

[35] K. Kalli, G. Simpson, K. Zhou, L. Zhang, D. Birkin, T. Ellingham, and I. Bennion, "Spectral modification of type IA fiber Bragg gratings by high power near infra-red lasers," Meas. Sci. Technol., vol. 17, no. 5, pp. 968-974, 2006.

[36] G. N. Smith, K. Kalli, and K. Sugden "Advances in femtosecond micromachining and inscription of micro and nano photonic devices," in Frontiers in Guided Wave Optics and Optoelectronics, Bishnu Pal. Ed. InTech, Croatia, 2010, pp. 674.

[37] G. N. Smith, K. Kalli, I. Bennion, and K. Sugden, "Demonstration of inscription and ablation of phase masks for the production of 1st, 2nd, and 3rd order FBG gratings using a femtosecond laser," in SPIE, Advanced Fabrication Technologies for Micro/Nano Optics and Photonics II, vol. 7205, pp 720511, 2009.

[38] T. Geernaert, K. Kalli, C. Koutsides, M. Komodromos, T. Nasilowski, W. Urbanczyk, J. Wojcik, F. Berghmans, and H. Thienpont, "Point-by-point fiber Bragg grating inscription in free-standing step-index and photonic crystal fibers using near-IR femtosecond laser," Opt. Lett., vol. 35, no. 10, pp. 1647-1649, 2010.

[39] K. T. O'Mahoney, A. S. Main, D. J. Webb, A. Martinez, and D. A. Flavin, "Implications of high power losses in IR femtosecond laser inscribed fiber Bragg gratings," in SPIE, Reliability of Optical Fiber Components, Devices, Systems, and Networks III, vol. 6193, pp. 61930Z, 2006.

[40] N. Jovanovic, J. Thomas, R. J. Williams, M. J. Steel, G. D. Marshall, A. Fuerbach, S. Nolte, A. Tünnermann, and M. J. Withford, "Polarization-dependent effects in point-by-point fiber Bragg gratings enable simple, linearly polarized fiber lasers," Opt. Exp., vol. 17, no. 8, pp. 6082-6095, 2009

[41] K. Kalli, T. Allsop, C. Koutsides, E. Davies, D. Webb, and L. Zhang, "Femtosecond laser inscription of fiber Bragg gratings with low insertion loss and minor polarization dependence," presented at Bragg Gratings, Photosensitivity, and Poling in Glass Waveguides (BGPP), Karlsruhe, Gernamy, 2010 (paper: BMA3).

[42] T. Erdogan, "Cladding-mode resonances in shortand long- period fiber grating filters," J. Opt. Soc. Am. A, vol. 14, no. 8, pp. 1760-1773, 1997.

[43] B. J. Eggleton, P. A. Krug, L. Poladian, and F. Ouellette, "Long periodic superstructure Bragg gratings in optical fibers," Elec.. Lett., vol. 30, no. 19, pp. 1620-1622, 1994.

[44] N. G.. R. Broderick and C. M. De Sterke, "Theory of grating superstructures," Phys. Rev. E, vol. 55, no. 3, pp. 3634-3646, 1997.

[45] H. Rao, R. Scarmozzino, and R. M. Osgood, “A bidirectional beam propagation method for multiple dielectric interfaces," IEEE Phot. Tech. Lett., vol. 11, no. 7 , pp. 830-832, 1999 .

[46] C. Koutsides, K. Kalli, D. J. Webb, and L. Zhang, "Characterizing femtosecond laser inscribed Bragg grating spectra," Opt. Exp., vol. 19, no. 1, pp. 342-352, 2011.

[47] T. Allsop, K. Kalli, K. Zhou, Y. Lai, G. Smith, M. Dubov, D. J. Webb, and I. Bennion, "Long period gratings written into a photonic crystal fiber by a femtosecond laser as directional bend sensors," Opt. Comm., vol. 281, no. 20, pp. 5092-5096, 2008.

[48] H. Dobb, K. Kalli, and D. J. Webb, "Measured sensitivity of long period gratings in photonic crystal fiber," Opt. Comm., vol. 260, no. 1, pp. 184-191, 2006.

[49] H. Dobb, K. Kalli, and D. J. Webb, “Temperature insensitive long period grating sensors in photonic crystal fiber," Elec. Lett., vol. 40, no. 11, pp. 657-658, 2004.

[50] J. S. Petrovic, D. J. Webb, V. Mezentsev, H. Dobb, K. Kalli, and I. Bennion, "Nondestructive index profiling of long period gratings in photonic crystal fibers," Opt. Quant. Elec., vol. 38, no. 9-11, pp. 913-920, 2006.

[51] J. S. Petrovic, V. Mezentsev, H. Dobb, D. J. Webb, K. Kalli, and I. Bennion, "Multiple period resonances in long period gratings in photonic crystal fibers," Opt. Quant. Elec., vol. 38, no. 1-3, pp. 209-216, 2006.

[52] J. S. Petrovic, H. Dobb, V. Mezentsev, K. Kalli, D. J. Webb, and I. Bennion, "Sensitivity of LPGs in PCFs fabricated by an electric arc to temperature, strain, and external refractive index," IEEE J. Lightwave Tech., vol. 25, no. 5, pp. 1306-1312, 2007.

[53] W. Xin, C. Shuying, D. Zhigang, W. Xiaoyang, S. Changhai, and C. Jianping, "Experimental study of some key issues on fiber-optic interferometric sensors detecting weak magnetic field," IEEE Sens. J., vol. 8, no. 7, pp. 1173-1179, 2008. 
[54] L. Sun, S. Jiang, and J. R. Marciante, "All-fiber optical magnetic-field sensor based on Faraday rotation in highly terbium-doped fiber," Opt. Exp., vol. 18 , no. 6, pp. 5407-5412, 2010.

[55] P. D. Dinev, "A two-dimensional remote fiber-optic magnetic field and current sensor," Meas. Sci. Tech., vol. 7, no. 9, pp. 1233-1237, 1996.

[56] G. N. Smith, T. Allsop, K. Kalli, C. Koutsides, R. Neal, K. Sugden, P. Culverhouse, and I. Bennion, "Characterisation and performance of a terfenol-D coated femtosecond laser inscribed optical fiber Bragg sensor with a laser ablated microslot for the detection of static magnetic fields," Opt. Exp., vol. 19, no. 1, pp. 363-370, 2011.

[57] K. Kalli, H. L. Dobb, D. J. Webb, K. Carroll, M. Komodromos, C. Themistos, G. D. Peng, Q. Fang, and I. W. Boyd, "Electrically tunable Bragg gratings in single mode polymer optical fiber," Opt. Lett., vol. 32, no. 3, pp. 214-216, 2007.

[58] K. Kalli, H. L. Dobb, D. J. Webb, C. Themistos, M. Komodromos, G. D. Peng, Q. Fang, and I. W. Boyd, "Development of electrically tunable Bragg grating filter operating at $1.55 \mu \mathrm{m}$ in single mode polymer optical fiber," Meas. Sci. Tech., vol. 18, no. 10, pp. 3155-3164, 2007.

[59] H. Dobb, D. J. Webb, K. Kalli, A. Argyros, M. C. J. Large, and M. A. van Eijkelenborg, "Continuous wave ultraviolet light-induced fiber Bragg gratings in few- and single-mode microstructured polymer optical fibers," Opt. Lett., vol. 30, no. 24, pp. 3296-3298, 2005.

[60] K. E. Carroll, C. Zhang, D. J. Webb, K. Kalli, A. Argyros, and M. C. J. Large, "Thermal response of Bragg gratings in PMMA microstructured optical fibers," Opt. Exp., vol. 15, no. 14, pp. 8844-8850,
2007.

[61] I. P. Johnson, K. Kalli, and D. J. Webb, "827 nm Bragg grating sensor in multimode microstructured polymer optical fiber," Elec. Lett., vol. 46, no. 17, pp. 1217-1218, 2010.

[62] I. P. Johnson, W. Yuan, A. Stefani, K. Nielsen, H. K. Rasmussen, L. Khan, D. J. Webb, K. Kalli, and O. Bang, "Optical fiber Bragg grating recorded in TOPAS cyclic olefin copolymer," Elec. Lett., vol. 47, no. 4, pp. 271-272, 2011.

[63] X. Chen, C. Zhang, D. J. Webb, K. Kalli, and G. D. Peng, "Highly sensitive bend sensor based on Bragg grating in eccentric core polymer fiber," IEEE Phot. Tech. Lett., vol. 22, no. 11, pp. 850-852, 2010.

[64] X. Chen, C. Zhang, D. J. Webb, G. D. Peng, and K. Kalli, "Bragg grating in polymer optical fiber for strain, bend and temperature sensing," Meas. Sci. Tech., vol. 21 no. 9, 2010.

[65] T. D. P. Allsop, R. Neal, E. Davies, C. Mou, P. Bond, S. Rehman, K. Kalli, D. J. Webb, P. Calverhouse, and I. Bennion, "Low refractive index gas sensing using a surface plasmon resonance fiber device," Meas. Sci. Tech., vol. 21, no. 9, 2010.

[66] T. Allsop, R. Neal, C. Mou, P. Brown, S. Saied, S. Rehman, K. Kalli, D. J. Webb, J. Sullivan, D. Mapps, and I. Bennion, "Exploitation of multilayer coatings for infrared surface plasmon resonance fiber sensors," Appl. Opt., vol. 48, no. 2, pp. 276-286, 2009.

[67] T. Allsop, R. Neal, C. Mou, P. Brown, S. Rehman, K. Kalli, D. J. Webb, D. Mapps, and I. Bennion, "Multilayered coated infra-red surface plasmon resonance fiber sensors for aqueous chemical sensing," Opt. Fib. Tech., vol. 15, no. 5-6, pp. 477-482, 2009. 